\title{
Scanning Tunneling Spectroscopy Study of Surface States of $3 d$ Metals: Chemical Identification, Magnetic Contrast and Orbital Kondo Resonance States
}

\author{
M.M.J. BischofF ${ }^{a}$, C.M. FAnG ${ }^{a}$, R.A. DE GroOT $^{a}$, \\ G.M.M. HeiJneN ${ }^{a}$, M.I. KATSNELSON ${ }^{a}$, \\ O.Yu. KolesnyChenKO ${ }^{a}$, R. DE KORT ${ }^{a}$, A.I. LiChtenstein ${ }^{a}$, \\ A.J. QuinN ${ }^{a}$, A.L. Vasquez DE Parma ${ }^{a, b}$, T.K. Yamada ${ }^{a, c}$ \\ AND H. VAN KEMPEN ${ }^{a, *}$ \\ ${ }^{a}$ NSRIM, University of Nijmegen, Toernooiveld 1 \\ 6525 ED Nijmegen, The Netherlands \\ ${ }^{b}$ Dpto. de Física de la Materia Condensada, Universidad Autónoma de Madrid \\ 28094 Madrid, Spain \\ ${ }^{c}$ Faculty of Science, Gakushuin University, 171-8588 Mejiro, Tokyo, Japan \\ The surface states of $3 d$ transition metals are studied by scanning tun- \\ neling microscopy and scanning tunneling spectroscopy. The results show \\ that surface states can be used for chemical identification with high spatial \\ resolution. The spin polarized nature of the surface states allows us to ob- \\ tain magnetic contrast in scanning tunneling miroscopy imaging with near \\ atomic resolution. For $\mathrm{Cr}(100)$ we found that the surface state close to the \\ Fermi level can be explained by an orbital Kondo resonance surface state. \\ PACS numbers: 73.20.-r, 68.37.-d, 75.70.-i
}

\section{Introduction}

Surface states of $3 d$ metals play an important role in catalysis and crystal growth. In addition it has been shown that surface states can be used for local chemical identification of elements in scanning tunneling microscopy (STM) studies [1]. Also these states can be spin polarized and so can contribute strongly to spin polarized tunneling currents which is important for spin electronic devices

*corresponding author; e-mail: hvk@sci.kun.nl 
and magnetic imaging. In this paper we will discuss: (i) How local is the local chemical identification; (ii) The use of the surface state for magnetic imaging; (iii) The nature of the surface state, especially for Cr.

\section{Chemical identification}

Davies et al. [1] showed by STM and scanning tunneling spectroscopy (STS) measurements that the surface states of $\mathrm{Fe}(100)$ and $\mathrm{Cr}(100)$ occur at different energies and so can be used for local chemical identification of the element to which the tunneling current flows. The questions we address in this paper are: How local is the local chemical identification? Is one monolayer of a guest element sufficient to suppress the surface state of the host metal? How sharp is the boundary between two regions with different surface state properties? To this end we studied $\mathrm{C}$ and $\mathrm{O}$ impurities on $\mathrm{V}(100)$ [2] and $\mathrm{Fe}(100)$, respectively, pure overlayers of $\mathrm{Au}$ on $\mathrm{Fe}(100)$ [3], and multilayers of $\mathrm{Mn}$ on $\mathrm{Fe}(100)$ [4-6]. In addition also the influence of atomic steps on $\mathrm{Fe}, \mathrm{Cr}$, and $\mathrm{V}$ have been studied. In this paper some examples of the results will be given.

The experiments were performed in a UHV system with a base pressure in the low $10^{-11}$ mbar range [3]. During deposition of $\mathrm{Mn}$ and Au on the substrate (iron whiskers) the pressure was kept in the low $10^{-10}$ mbar. We used an Omicron STM, type UHV STM-1. For the STS observations usually at every pixel of the image a current $(I)$ versus bias voltage $(V)$ curve was measured. The derivative $\mathrm{d} I / \mathrm{d} V$ was obtained by numerical differentiation. In many cases the signal quality was sufficient to fit the observed surface state peaks in the $\mathrm{d} I / \mathrm{d} V$ versus $V$ curves to a Gaussian function at every pixel of the image. In this way peak energy, peak width, and peak height images could be obtained around impurities and other defects.

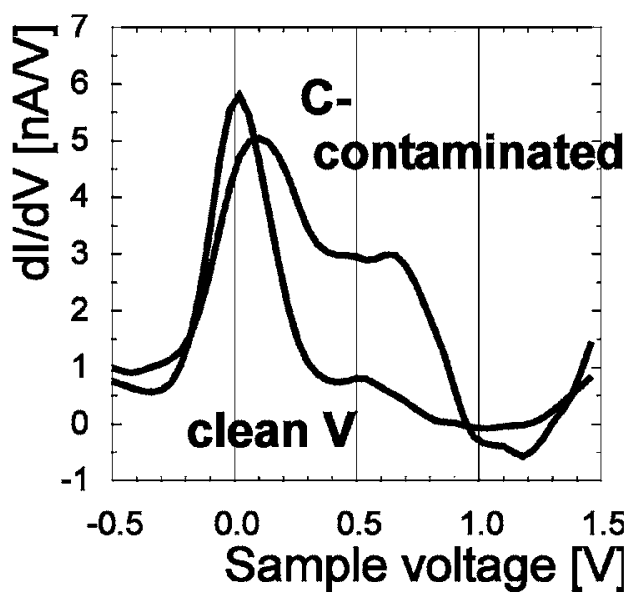

Fig. 1. $\mathrm{d} I / \mathrm{d} V$ versus bias voltage $(V)$ of clean and C-contaminated vanadium (100) surface. 


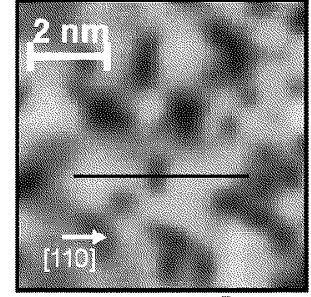

topography $(-0.3 \mathrm{~V}, 1 \mathrm{nA})$

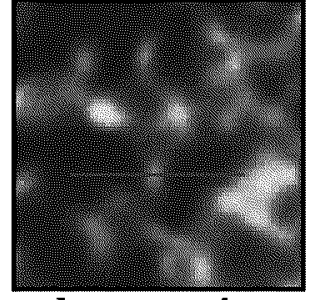

peak energy image (Gaussian fits)

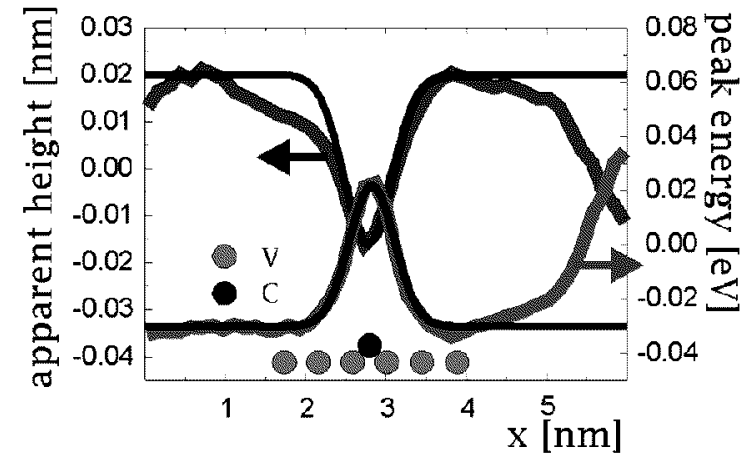

Fig. 2. Upper left panel shows the topography of a carbon contaminated V(100) surface. The right panel shows a peak energy image; the gray scale ranges from $-0.03 \mathrm{eV}$ (black) to $0.1 \mathrm{eV}$ (white). The lower panel gives the line profiles indicated in the upper panels.

Figure 1 shows an STS measurement of V, slightly contaminated by 0.05 monolayer (ML) of carbon. A clear shift of the surface state peak of vanadium is seen. Clean vanadium shows a strong surface state peak at $0.03 \mathrm{eV}$ below the Fermi level. At the carbon contaminated surface the peak energy, peak height, and peak width are all changed. To study the local character of the influence of the carbon impurity a comparison is made between the height map of the sample and the peak energy map (Fig. 2). From other experiments it is known already that the carbon shows up in the height map as a suppression. From the cross sections through the contamination, consisting of one or perhaps two carbon atoms, one can see that the spatial resolution in the height map and the peak energy map is about the same, with a FWHM of $0.8 \mathrm{~nm}$.

Similar experiments have been performed for oxygen on $\mathrm{Fe}(100)$. An example is given in Fig. 3. A single oxygen impurity shifts the surface state peak energy to a higher energy by $0.04 \mathrm{eV}$. The peak energy map shows again a spatial resolution equal to spatial resolution in the height map $(\sim 1.0 \mathrm{~nm})$. This resolution is about what has to be expected at the setpoint used in the STS measurements. This setpoint corresponds to a quite large tip-sample distance needed to be able to extend the $I \sim V$ measurements to quite high voltages. Also for atomic steps, 


\section{$\mathrm{Fe}(001)+\mathrm{O}$}

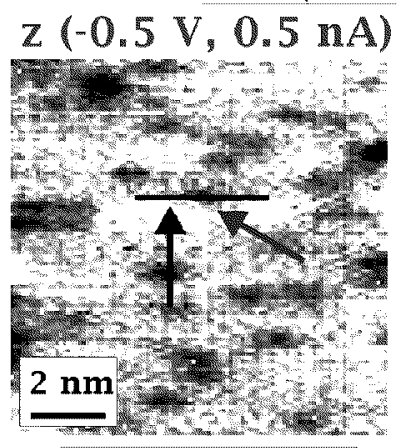

peak energy

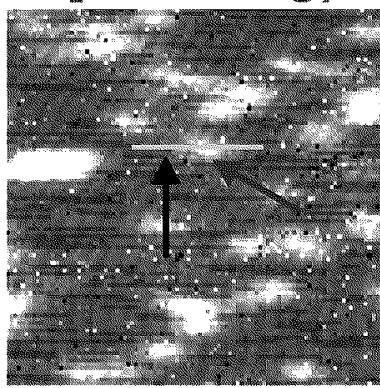

$+0.04 \mathrm{eV}$ at $\mathrm{O}$
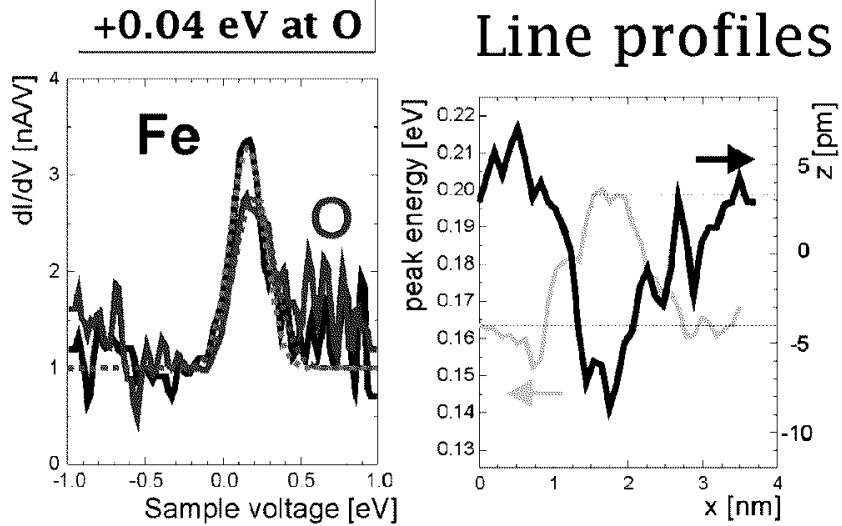

Fig. 3. The upper panels show the height image and peak energy image of oxygen impurities on $\mathrm{Fe}(100)$. The lower left panel shows the small shift of $0.04 \mathrm{eV}$ of the peak energy at an oxygen impurity. The arrows in the upper panels show the positions of the measurements drawn in the lower left panel. The lower right panel gives the line profiles indicated in the upper panels.

which suppress the surface state completely in $\mathrm{Fe}(100)$ and $\operatorname{Cr}(100)$ (but not in $\mathrm{V}(100)$ ), a similar spatial resolution of 0.7 to $1.0 \mathrm{~nm}$ was obtained in the STM and STS measurements.

Another interesting question is how many contamination atoms are needed to suppress the surface state. Figure 4 shows that a monolayer of pure $\mathrm{Au}$ on $\mathrm{Fe}(100)$ suppresses the surface state completely. By comparing the sharpness of the edges of the gold islands, observed in the height observation and in the electronic structure observation, we can conclude that the change in the electronic structure is very abrupt. The edges of the islands show up in the STM map and STS map with similar resolution as seen before for the single impurities. Also for Mn monolayer islands on $\mathrm{Fe}(100)$ the same abruptness in the change of electronic structure has been observed. In this case the peak energy shifts from the iron value of $0.17 \mathrm{eV}$ to $0.35 \mathrm{eV}$ for the $\mathrm{Mn}$ monolayer [3]. 

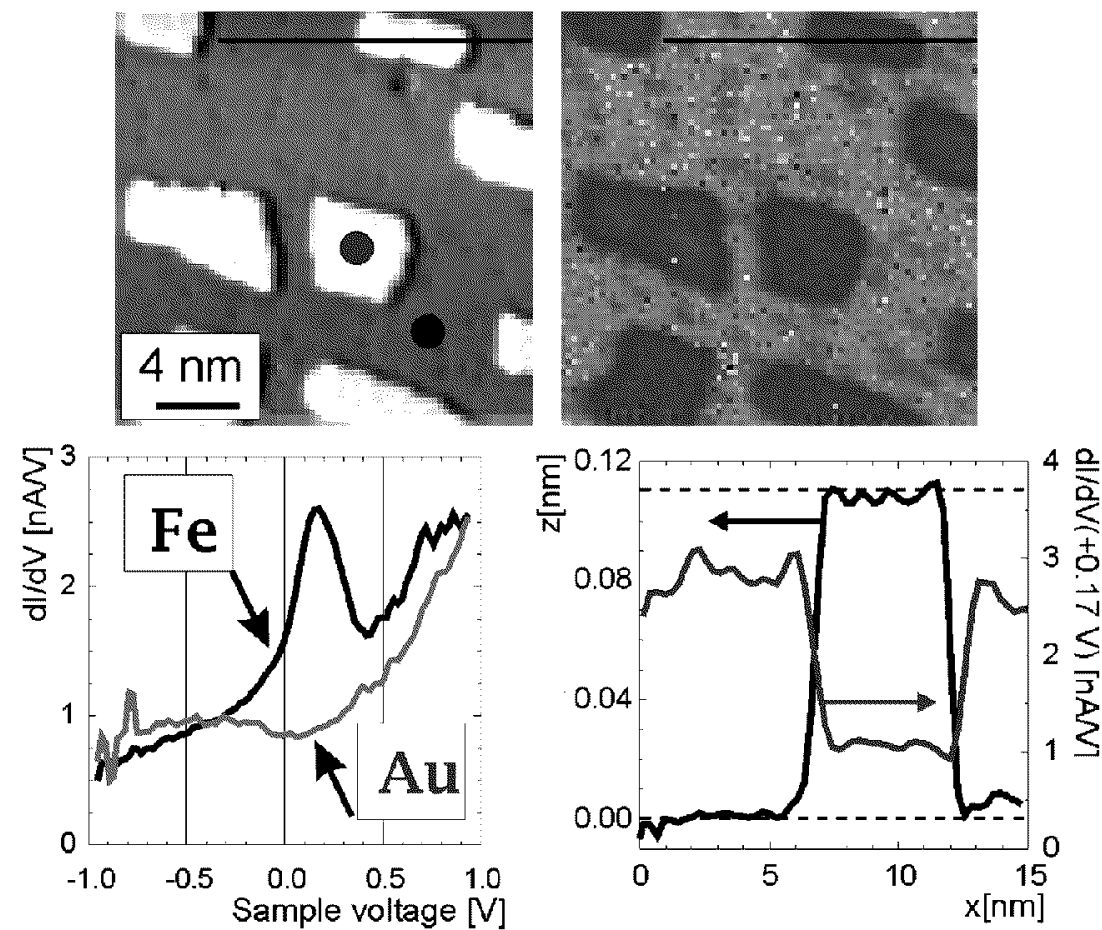

Fig. 4. The upper left panel gives the height image of gold islands on Fe(100) taken at a setpoint of $-0.5 \mathrm{~V}$ and $0.6 \mathrm{nA}$. The upper right panel gives the $\mathrm{d} I / \mathrm{d} V$ map at $0.17 \mathrm{~V}$. The left lower panel shows the suppression of the iron surface state by a gold monolayer. The positions of these measurements are indicated in the upper left panel by dots. The lower right panel gives the line profiles indicated in the upper panels.

All of the described experiments show that the spatial resolution of the observation of the surface state is comparable to the resolution in the height images. The resolution obtained is $0.7 \mathrm{~nm}$ in the best cases. This resolution can be improved when the tip-sample distance can be decreased. However for the STS measurements this distance is usually quite large because otherwise the current should increase too much at the higher voltage end of the $I \sim V$ measurements. But for surface states close to the Fermi level the voltage can be kept small and some improvement in resolution can still be expected.

\section{Magnetic imaging}

The surface state of the $3 d$ metals can be spin polarized to a high degree and so can contribute strongly to spin polarized tunneling currents. Magnetic contrast in STS can be expected when the sample has domains with different magnetization directions and the STM tip is magnetized. 
An interesting sample is Mn/Fe(100) (Fig. 5). Previous SEMPA experiments in the NIST laboratory [7] have shown that the Mn sample consists of ferromagnetic layers, which are antiferromagnetically stacked. As a result a sharp boundary between domains with opposite magnetization direction might be present at every single atomic step at the surface. We studied in detail the growth conditions to get samples with nearly layer by layer growth and a limited amount of interface diffusion $[4,6]$. By choosing the right growth conditions we obtained samples where a number of Mn layers are exposed, see Fig. 6. Figure 6 has been recorded with a W tip. All Mn layers from the fourth layer up, show the same electronic structure and so no specific features are observed in the $\mathrm{d} I / \mathrm{d} V$ map.

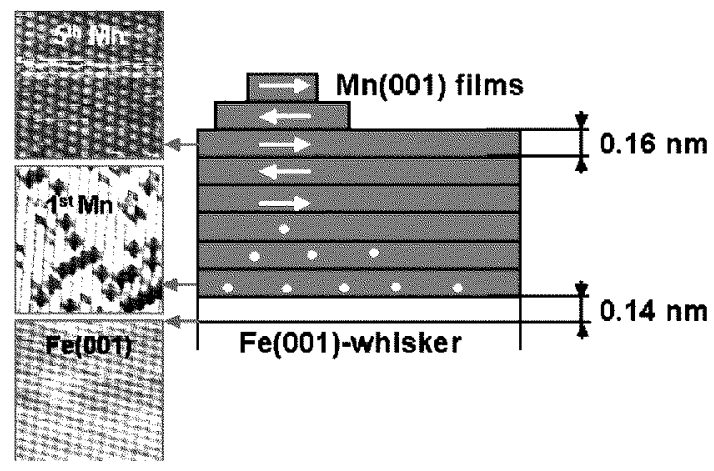

Fig. 5. Schematic drawing of a Mn sample grown on a Fe whisker (right panel). The left panels give chemically resolved images of the indicated layers showing the iron contamination in the first Mn layer as black spots.

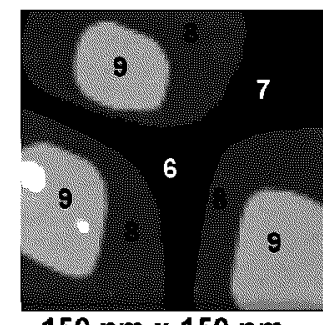

$150 \mathrm{~nm} \times 150 \mathrm{~nm}$
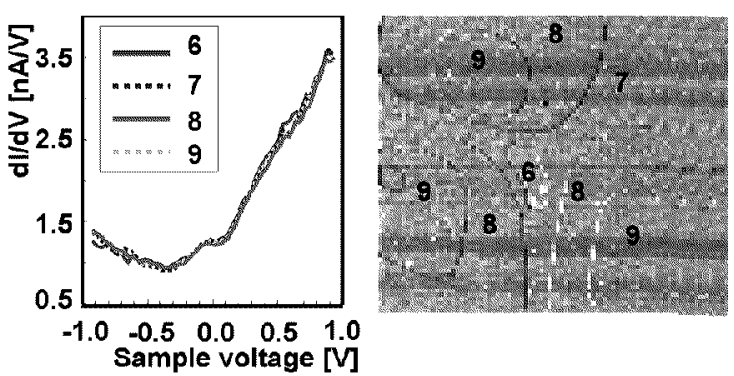

Sample voltage $[\mathrm{V}]$

Fig. 6. Height image for 7.2 ML of Mn on Fe (left). Layer 6 to 10 are exposed (setpoint $0.5 \mathrm{~V}, 0.5 \mathrm{nA}$ ). Spectroscopy (middle) and $\mathrm{d} I / \mathrm{d} V$ map at $0.2 \mathrm{~V}$ (right). Measurements are taken with a $\mathrm{W}$ tip.

To get a ferromagnetic tip we use a $W$ tip, covered by a thin layer of iron of 7-10 nm following the method described in Ref. [8]. Before the iron is evaporated on the tip, the tip is blunted by heating by electron bombardment to $\sim 2000^{\circ} \mathrm{C}$. Field emission spectroscopy shows that the tip radius is larger than $300 \mathrm{~nm}$. As 
the magnetization of the thin iron layer is expected to follow the direction of the layer the magnetization of the tip will be parallel to the sample surface.

Figure 7 shows that with the iron coated tip the even and odd layers show different electronic structures, corresponding to the different density of states for the spin up and spin down directions relative to the tip magnetization direction. A clear magnetic contrast at the atomic steps is observed. As in our preparation procedure the tip magnetization direction is arbitrary, there is some chance that the magnetization direction of tip and sample are perpendicular and no contrast is observed. In that case we can change the tip magnetization direction by applying a bias voltage pulse of appropriate strength and duration [9].

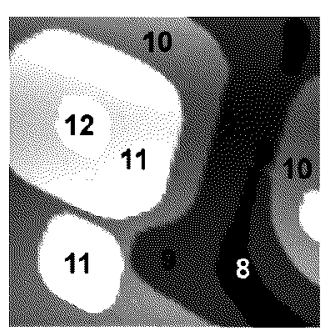

$100 \mathrm{~nm} \times 100 \mathrm{~nm}$
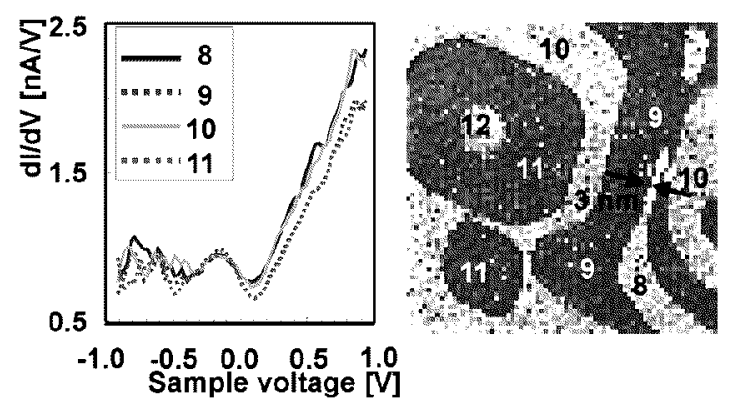

Fig. 7. Height image for 9.5 ML of Mn on Fe (left). Layer 8 to 12 are exposed. Spectroscopy (middle) and $\mathrm{d} I / \mathrm{d} V$ map at $0.2 \mathrm{~V}$ (right). Clearly the difference between even and odd layers can be seen. Note that also in the narrow trench $(3 \mathrm{~nm})$ the magnetic contrast is well resolved.

To find the ultimate magnetic spatial resolution the atomic steps are not suitable because the admixture of height information and magnetic information might hamper a proper analysis. However at steps in the iron whisker one can have an even and odd number of Mn layers meeting each other nearly without a step in the sample surface height (see Fig. 8). Using such a hidden iron step we found a spatial resolution in the magnetic contrast of $0.4 \mathrm{~nm}$ in the best cases.

To get quantitative information about the degree of spin polarization of the sample is not straight forward. One quantitative measure is the asymmetry in $\mathrm{d} I / \mathrm{d} V$ defined as

$$
A_{(\mathrm{d} I / \mathrm{d} V)}=[(\mathrm{d} I / \mathrm{d} V) \uparrow \uparrow-(\mathrm{d} I / \mathrm{d} V) \uparrow \downarrow] /[(\mathrm{d} I / \mathrm{d} V) \uparrow \uparrow+(\mathrm{d} I / \mathrm{d} V) \uparrow \downarrow] .
$$

However, as can be seen in Fig. 9, the results vary strongly for different setpoints and also for different tips (not indicated in the figure). The reason behind this phenomenon is that $\mathrm{d} I / \mathrm{d} V$ is not directly proportional to the density of states but to the density of states times an energy dependent tunneling probability $T$. A more appropriate asymmetry factor could be 


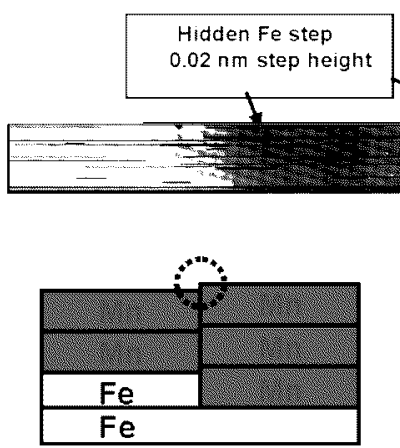

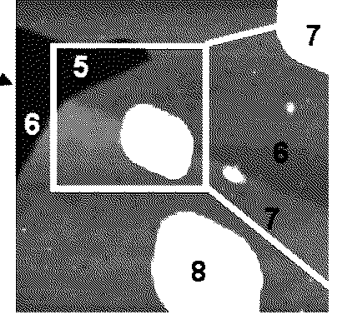

$150 \mathrm{~nm} \times 150 \mathrm{~nm}$ $(-0.6 \mathrm{~V}, 0.5 \mathrm{nA})$

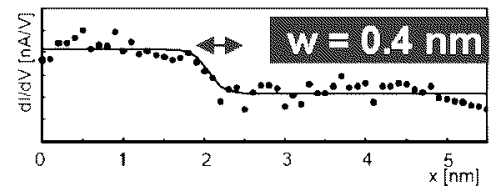

Fig. 8. Spatial resolution in magnetic contrast above a hidden iron step (setpoint $-0.6 \mathrm{~V}, 0.5 \mathrm{nA})$.
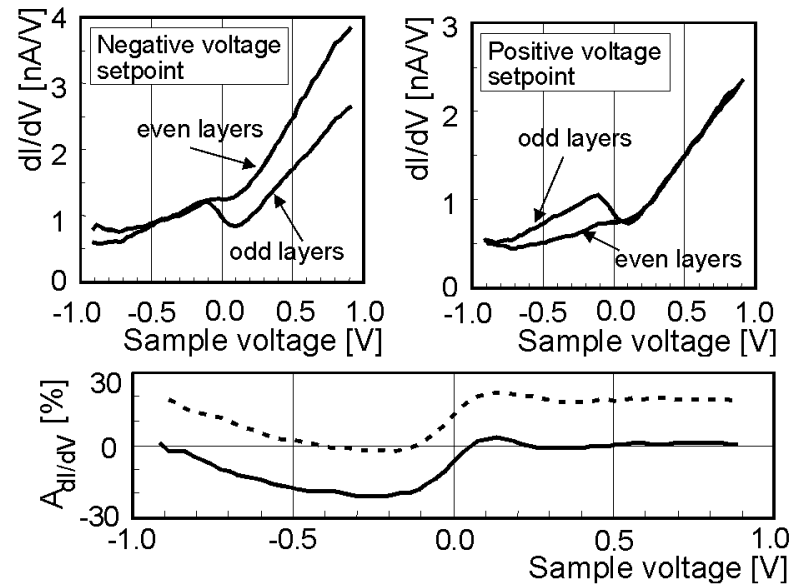

Fig. 9. Different $\mathrm{d} I / \mathrm{d} V$ results obtained for different setpoints (upper panels). Lower panel: The asymmetry factor obtained for different setpoints: Dashed line for negative voltage setpoint; drawn line for positive voltage setpoint.

$$
A_{(\mathrm{d} I / \mathrm{d} V) / T}=\frac{\{[(\mathrm{d} I / \mathrm{d} V) / T] \uparrow \uparrow-[(\mathrm{d} I / \mathrm{d} V) / T] \uparrow \downarrow\}}{\{[(\mathrm{d} I / \mathrm{d} V) / T] \uparrow \uparrow+[(\mathrm{d} I / \mathrm{d} V) / T] \uparrow \downarrow\}} .
$$

The crucial problem with this approach is how to find $T$. We used an approximation proposed by Ukraintsev [10]. By fitting the measured $\mathrm{d} I / \mathrm{d} V$ as a function of bias voltage $T$ can be obtained. As can been seen in Fig. 10, at positive bias voltage, where the sample density of states dominates, the asymmetry factor becomes tip and setpoint independent. At negative bias voltage, where the tip density of states dominates, the scattering in the data is still large for different tips as can be expected. Applying the correction for the energy dependence of $T$ also results in 


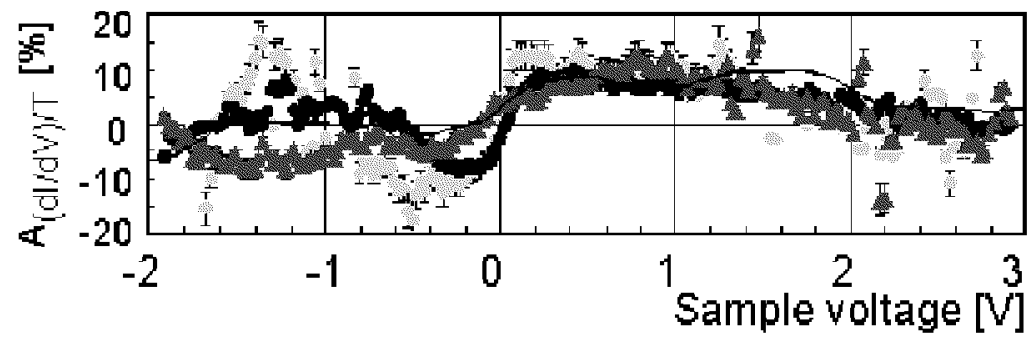

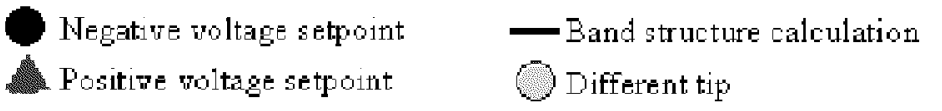

Fig. 10. The asymmetry factor corrected for the tunneling probability $T$. The drawn line indicates the result of band structure calculation.

a clear reproducible observation of a broad density of state peak at $0.8 \mathrm{eV}$. This peak can be explained by a combination of spin polarized $d_{z^{2}}$ states found in band structure calculations performed at our institute [5].

\section{Surface state of $\mathrm{Cr}(100)$}

The experiments on $\mathrm{Cr}(100)$ were performed at $4.2 \mathrm{~K}$ with a low temperature STM built in our institute [11]. The sample surface was prepared by in situ cleaving

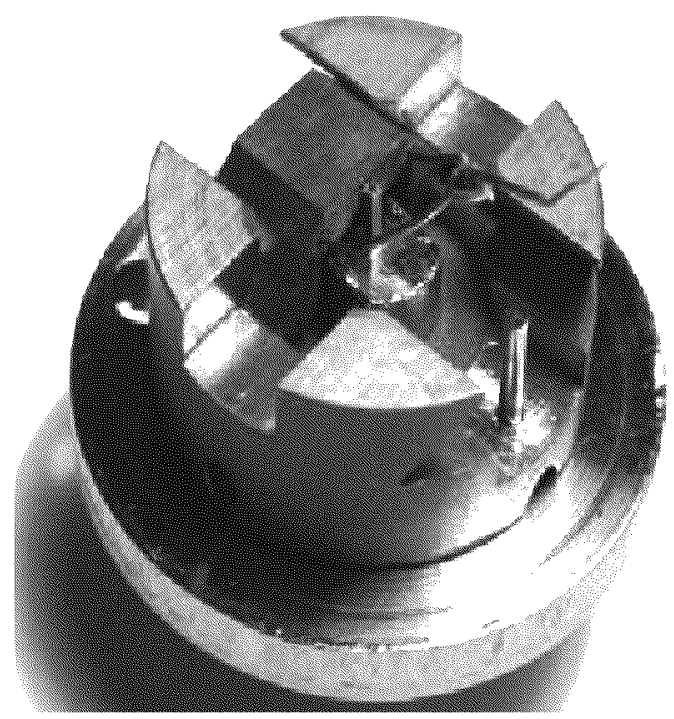

Fig. 11. 4.2 $\mathrm{K}$ cleave stage. The matchstick shaped $\mathrm{Cr}$ sample is visible in the middle. A wire pulls the "sledge", visible at the left, to the right till the sample breaks. 


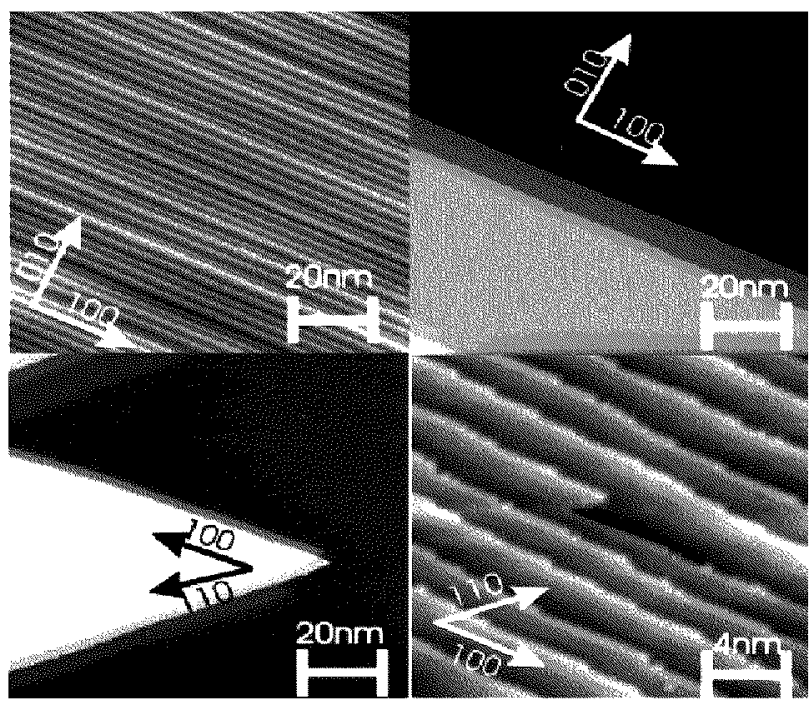

Fig. 12. Different terrace structures observed on cleaved Cr samples.
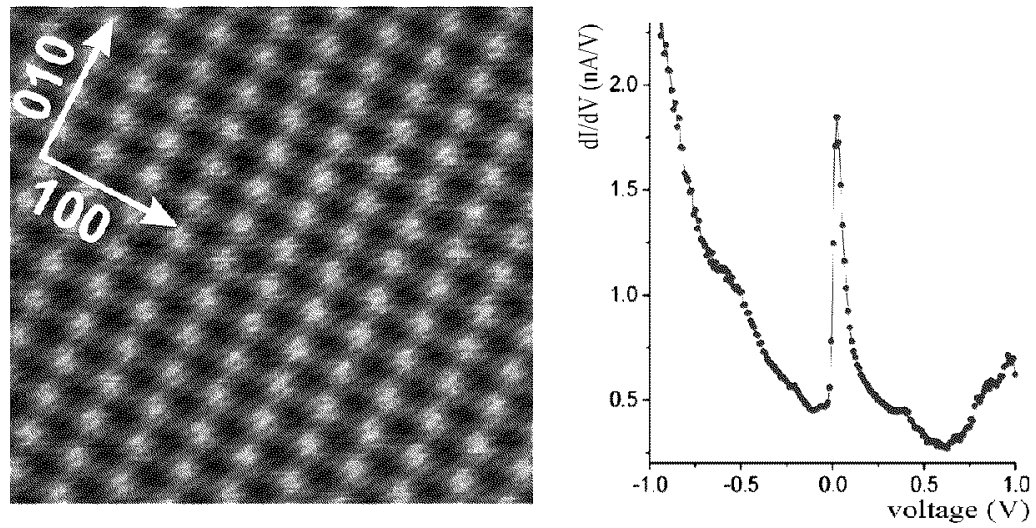

Fig. 13. The right panel shows the $\mathrm{d} I / \mathrm{d} V$ peak observed on an atomically flat terrace shown on the left $(3 \times 3 \mathrm{~nm})$. The atomic corrugation of $0.0015 \mathrm{~nm}$ is clearly visible in the left panel.

at $4.2 \mathrm{~K}$ of a $99.999 \%$ purity single crystal of $\mathrm{Cr}$. The cleavage of a $\mathrm{Cr}$ single crystal can produce (with some luck) an atomically flat and clean (100) surface. Figure 11 shows the sample cleavage stage. Figure 12 shows some typical examples of different surface structures, which can be obtained ranging from very narrow terraces to large flat terraces. However the cleaving can also result in quite rough terraces as shown in the lower right panel of Fig. 12 or in very rough surfaces (not shown). Spectroscopic measurements on large monoatomic terraces (shown on the left panel of Fig. 13) show a very sharp peak close to the Fermi level. 
Occasionally the Cr crystal contains impurities. Figure 14 shows the image of such impurities obtained at different bias voltages. Away from the peak energy the impurity shows up as a localized feature. However at the peak energy the impurity shows up as an extended feature with a cross like symmetry.
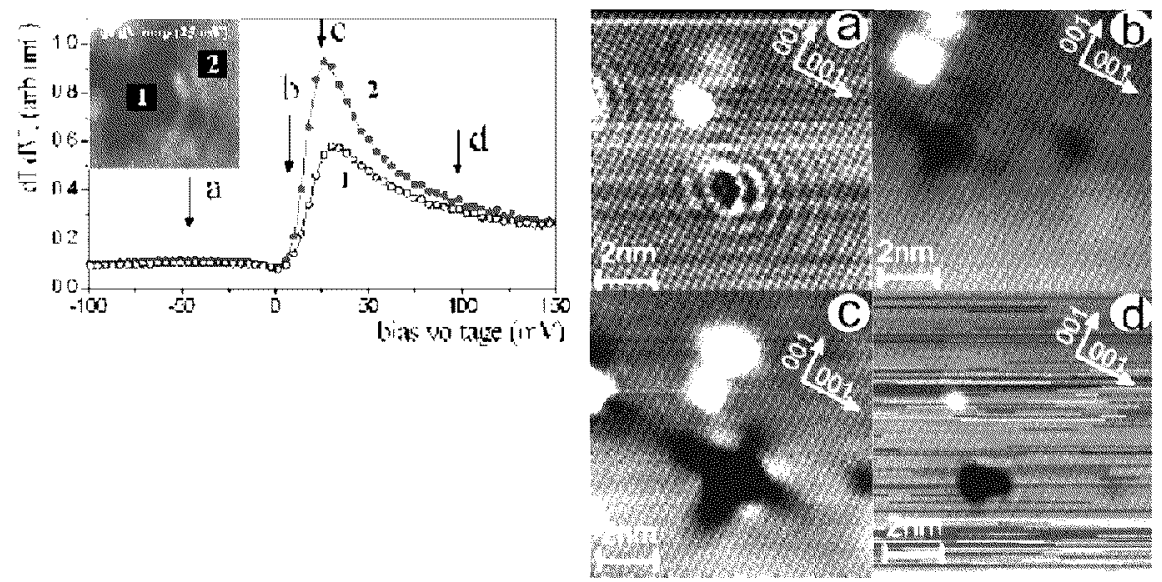

Fig. 14. The right panels show the observations of some impurities. The images are taken at the voltages indicated in the left panel. The inset in the left panel gives a $\mathrm{d} I / \mathrm{d} V$ map taken at $25 \mathrm{meV}$. The curves 1 and 2 are measured at the points indicated in the inset of the left panel.

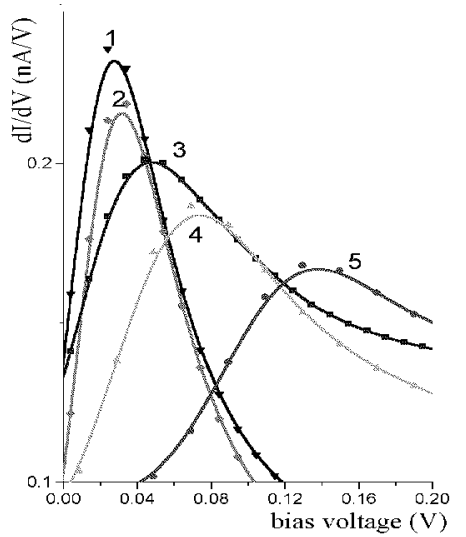

Fig. 15. Influence of the terrace width on the peak height and energy. The peaks 1 to 5 are measured at terraces of $20,5,2,1.5,0.8 \mathrm{~nm}$, respectively and have a position at 24 , $34,54,70$, and $130 \mathrm{mV}$.

It is difficult to explain the sharpness of the peak, the nonlocal influence of the impurities and the cross-like symmetry for a surface state with $d_{z^{2}}$ symmetry. An alternative explanation has been proposed based on the Kondo effect [12]. 
Tight binding mean field calculations [13] showed the existence of two degenerate spin-split $d_{x z}, d_{y z}$ surface states localized about $1 \mathrm{eV}$ above and below the Fermi level. The spatial symmetry of these states at the (100) surface together with the interaction with the conduction electrons can lead to the formation of a many-body Kondo resonance near the Fermi level. In this way an orbital singlet can be formed with the $d_{x z}, d_{y z}$ states mixed symmetrically similar to the spin-flip mechanism in the conventional Kondo effect [14].

The conventional Kondo effect is very sensitive to symmetry breaking of the spins by magnetic fields. This effect cannot play a role in the antiferromagnetic Cr. However, the (100) symmetry can be broken by atomic steps at the edges of the terraces at the surface. Figure 15 shows the influence of the width of the terraces on the surface state peak height and width. Indeed at narrow terraces the peak collapses.

Concluding, this works shows that the orbital degeneracy of the $d_{x z}, d_{y z}$ surface states can lead to the formation of an orbital Kondo resonance on $\operatorname{Cr}(100)$.

\section{Acknowledgment}

This work was supported by the Stichting voor Fundamenteel Onderzoek der Materie (FOM), which is funded by the Nederlandse Organisatie voor Wetenschappelijk Onderzoek (NWO), and by the European project MAGNETUDE. A.L.V.P. thanks the Nederlandse Organisatie voor Wetenschappelijk Onderzoek and the Ministerio de Ciencia y Technología for financial support. The authors are grateful to Dr. D.T. Pierce of the NIST laboratory for supplying the Fe whiskers. The work on $\mathrm{V}$ was done in cooperation with the group of Prof. P. Varga, TU Wien; we thank Prof. P. Varga for his kind hospitality to M.M.J.B.

\section{References}

[1] A. Davies, J. Stroscio, D.T. Pierce, R.J. Celotta, Phys. Rev. Lett. 76, 4175 (1996).

[2] M.M.J. Bischoff, C. Konvicka, A.J. Quinn, M. Schmid, J. Redinger, R. Podloucky, P. Varga, H. van Kempen, Surf. Sci. 513, 9 (2002); M.M.J. Bischoff, C. Konvicka, A.J. Quinn, M. Schmid, J. Redinger, R. Podloucky, P. Varga, H. van Kempen, Phys. Rev. Lett. 86, 2396 (2001).

[3] M.M.J. Bischoff, Ph.D. Thesis, University of Nijmegen, Nijmegen 2002.

[4] T.K. Yamada, M.M.J. Bischoff, T. Mizoguchi, H. van Kempen, Surf. Sci. 516, $179(2002)$.

[5] T.K. Yamada, M.M.J. Bischoff, G.M.M. Heijnen, T. Mizoguchi, H. van Kempen, Phys. Rev. Lett. 90, 056803 (2003).

[6] M.M.J. Bischoff, T. Yamada, A.J. Quinn, H. van Kempen, Surf. Sci. 501, 155 (2002).

[7] D.A. Tulchinsky, J. Unguris, R.J. Celotta, J. Magn. Magn. Mater 212, 91 (2000). 
[8] O. Pietzsch, A. Kubetzka, M. Bode, R. Wiesendanger, Science 292, 2053 (2001); R. Wiesendanger, M. Bode, Solid State Commun. 119, 341 (2001).

[9] T.K. Yamada, M.M.J. Bischoff, T. Mizoguchi, H. van Kempen, Appl. Phys. Lett. 82, 1437 (2003)

[10] V.A. Ukraintsev, Phys. Rev. B 48, 11176 (1996).

[11] J.W.G. Wildöer, A.J.A. van Roy, H. van Kempen, C.J.P.M. Harmans, Rev. Sci. Instrum. 65, 2849 (1994).

[12] O.Yu. Kolesnychenko, R. de Kort, M.I. Katsnelson, A.I. Lichtenstein, H. van Kempen, Nature 415, 507 (2002).

[13] L.E. Klebanov, R.H. Victoria, L.M. Falicov, D.A. Shirly, Phys. Rev. B 32, 1997 (1985).

[14] D.L. Cox, A. Zawadowski, Adv. Phys. 47, 599 (1998). 